\title{
Measurement of unsteady velocity flow field of pulsating flow
}

\author{
Petra Dančová, ${ }^{1,2, a}$, and Tomáš Vit ${ }^{1}$ \\ ${ }^{1}$ Technical University of Liberec, Studentská 2, 46117 Liberec 1, Czech Republic \\ ${ }^{2}$ Institute of Thermomechanics, CAS, v.v.i., Dolejškova 5, 18000 Praha, Czech Republic
}

\begin{abstract}
This paper describes an investigation of the commercial synthetic jet actuator "SynJet ZFlow 90 Outdoor Cooler" (Nuventix). The velocity flow field was investigated using the thermoanemometry method in the constant temperature mode. The Eigen frequency of the used actuator was also calculated in this work.
\end{abstract}

\section{Introduction}

This paper deals with an investigation of unsteady velocity flow field which generates the actuator of synthetic jet. The commercial actuator "SynJet ZFlow 90 Outdoor Cooler" from Nuventix Company is used for our research, [1].

What the term "synthetic jet" (SJ) means and how the SJ actuators work is described in works e.g. [2-4]. A SJ is synthesized by the interactions within a train of vortex rings or counter-rotating vortex pairs in axis-symmetric or two-dimensional geometry, [2]. These vortex rings are generated by the periodical motion of an actuator oscillating membrane. This membrane enables fluid suction into the actuator cavity in the first part of the membrane motion. Then in the second part of the period, this sucked fluid is exhausted from the actuator cavity. One of the main advantages of a SJ is that the time-mean mass flux of the oscillatory flow in the orifice is zero; hence the other common expression is a zero-net-massflux (ZNMF) jet. A ZNMF eliminates the requirement of a blower and piping for the fluid inlet. Though the SJ actuator works with a ZNMF at the orifice, the momentum of the resultant $\mathrm{SJ}$ at a specific distance from the lip in $y$ is non-zero [2-4].

\section{Experimental setup and method}

\subsection{Experimental setup}

For our experiments, the commercial synthetic jet actuator "SynJet ZFlow 90 Outdoor Cooler" (Nuventix) was used. For the schematic view and the actuator geometry see figure 1 , for the technical specification see [1]. The SJ actuator orifice has the outer and inner diameter of $88 \mathrm{~mm}$ and $85 \mathrm{~mm}$, respectively, and it is divided into four quarter circle parts (visible on figure 1 (c)). Figures 1 (c) and (d) also show the used coordinate system.

\footnotetext{
${ }^{\mathrm{a}}$ Corresponding author: petra.dancova@tul.cz
}
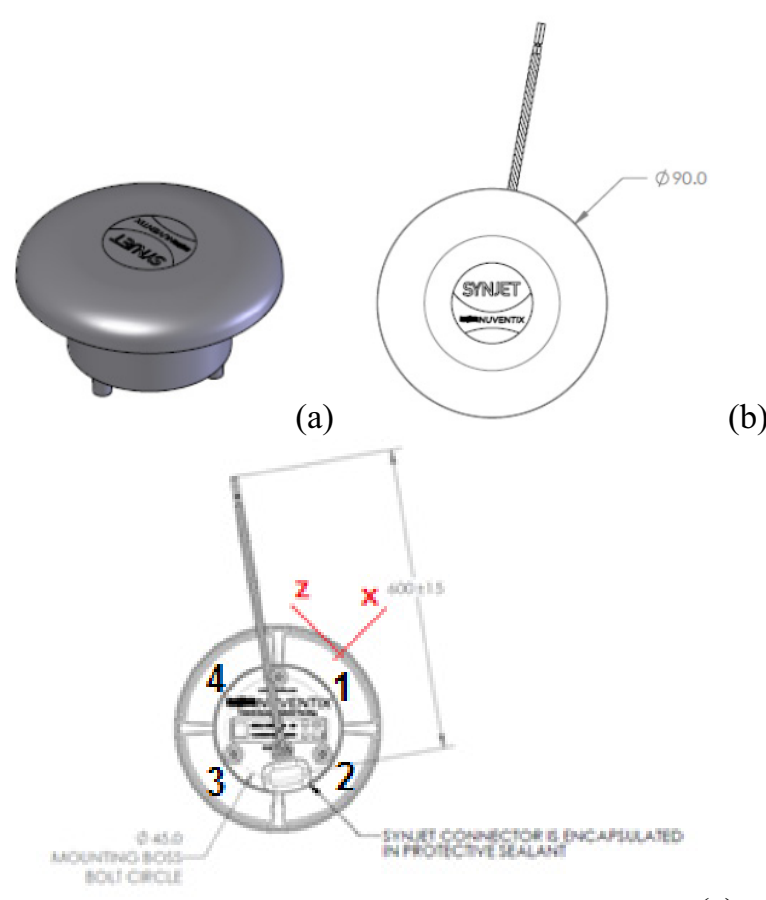

(b)

(c)

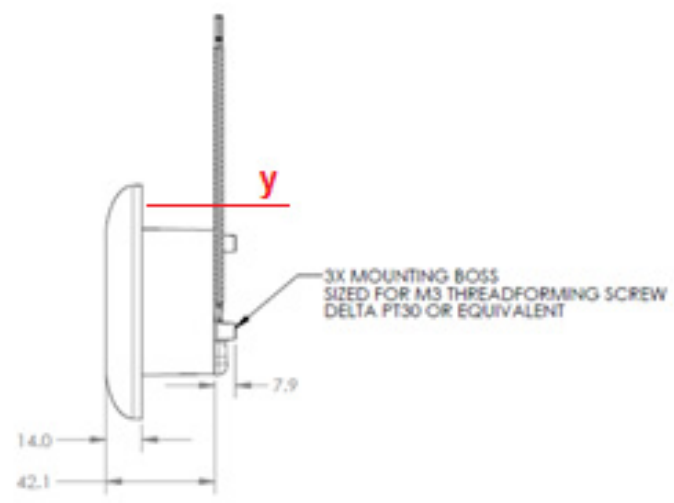

(d)

Figure 1. SynJet ZFlow 90 Outdoor Cooler, (a) schematic view, (b) view from the top, (c) platform, (d) side view. 


\subsection{Experimental method - HWA}

Hot wire anemometry (HWA) is one of the classical methods for measuring fluid velocities, including a fluctuating velocity component. It was used in this study to quantify SJ velocities in general and to evaluate the resonant frequency of SJ actuators in particular, [5].

The method is based on convective heat transfer from a heated body (hot wire or hot film probe) into a moving environment. The basic element of the measuring circuit is the probe, which is a wire fixed to the prongs of the carrier. The wire (typically tungsten) is heated by an electric current, [5]. The heat flux generated by the electric current in the wire element of length $d x$ can be formulated according to Joule's law in the form:

$$
d \dot{Q}_{\mathrm{J}}=\frac{I^{2} \chi_{\text {wire }}}{S_{\text {wire }}} d x
$$

where $I$ is the electric current, $\chi_{\text {wire }}$ is the specific resistance of wire material at the wire temperature $T_{\text {wire }}$ and $S_{\text {wire }}$ is the wire cross section area, [5].

The heat flux $d \dot{Q}_{\mathrm{J}}$ generated on the wire element $d x$ must be equal to the sum of the heat transferred into the surroundings, i.e. the heat transferred by convection $d \dot{Q}_{\text {conv }}$, the heat transferred by conduction into carriers $d \dot{Q}_{\text {cond }}$, the heat radiated into the surroundings $d \dot{Q}_{\mathrm{rad}}$, and the accumulated heat $d \dot{Q}_{\mathrm{ac}}$ :

$$
d \dot{Q}_{\mathrm{J}}=d \dot{Q}_{\mathrm{conv}}+d \dot{Q}_{\mathrm{cond}}+d \dot{Q}_{\mathrm{rad}}+d \dot{Q}_{\mathrm{ac}} .
$$

The heat dissipated by radiation is in most cases negligible, and the heat dissipated from the wire into the carrier can be considered as independent of the parameters of the flowing medium, see [5]. The convective heat flux $d \dot{Q}_{\text {conv }}$ transferred into the flowing medium can be formulated as:

$$
d \dot{Q}_{\text {conv }}=\pi d_{\text {wire }} \alpha\left(T_{\text {wire }}-T_{\infty}\right) d x,
$$

where $d_{\text {wire }}$ is the wire diameter, $\alpha$ is the heat transfer coefficient, $T_{\text {wire }}$ the wire surface temperature, and $T_{\infty}$ is the fluid temperature.

If a constant temperature (CT) mode is assumed (this mode is used for velocity measurement), the thermal balance for an infinitely long wire, where the heat transfer into the holder is ignored, is given by:

$$
I^{2} R_{\text {wire }}=\pi d_{\text {wire }} L_{\text {wire }} \alpha\left(T_{\text {wire }}-T_{\infty}\right),
$$

where $R_{\text {wire }}$ is the wire resistance on a heated wire with the temperature $T_{\text {wire }}$, and $L_{\text {wire }}$ is a length of the wire. After substitution, see [5], the equation (4) becomes:

$$
\frac{E_{\text {wire }}^{2}}{R_{\text {wire }}}=\left(A+B U^{n}\right)\left(T_{\text {wire }}-T_{\infty}\right) \text {, }
$$

where $A, B$ and $n$ are the functions of wire diameter and the material properties of the wire, and of the surrounding fluid, whose magnitude are determined through calibration, [5]. E is the voltage measured on the wire.
Overheat ratio: An important parameter of probes working in a CT regime is the so-called "overheat ratio":

$$
a_{\mathrm{h}}=\frac{R_{\mathrm{wire}}}{R_{\infty}}
$$

and it is a guide for adjusting the wire temperature. For velocity measurement, it is recommended select an overheat ratio as high as possible (maximum values are limited by the material properties of the wire, especially by a tendency toward faster corrosion at higher temperatures). For experiments in fluids, the overheat ratio is limited by the boiling temperature of fluid.

Velocity calibration: Calibration is used to determine the constants in established calibration relations for velocity measurement $E=f(U)$. The most common relations for HWA calibration are described in [5]. For experiments published in this paper, the polynomial curve fit was used:

$$
U=A E^{0}+B E^{1}+C E^{2}+D E^{3}+F E^{4},
$$

where $A, B, C, D$, and $F$ are the calibration constants, and $E$ is the voltage measured on the wire.

For experiments, the DANTEC system was used: two hot-film probes 55P11, anemometer 90C10, bus bar NI_CA1000, and A/D converter NI-PCI-MIO-16E-1. The measured data was analyzed in StreamWare 3.01 and Microsoft Excel.

\section{Experimental results}

The measurement was carried out with sampling frequency and number of samples $8 \mathrm{kHz}$ and 16384, respectively. Overheat ration and the temperature on the probes wire were set as 0.80 , and $230 \mathrm{~K}$, respectively.

For the measurement it was necessary to use two hotfilm probes 55P11; first was used as the reference (stable) probe and the second one (measuring probe) was used to receive data from the experiments. Because the reference probe was on the same position during whole measurement, the received signal on this probe was always the same. This is the reason why the signal from the reference probe was used for phase averaging of the signal received on the measuring probe. Principle of phase averaging can be found in [5 and 6].

Velocity calibration: The calibration constants were determined from forth order polynomial curve as:

$U=51.3514 E^{0}-114.3149 E^{1}+103.3519 E^{2}-$ $47.3964 E^{3}+9.4725 E^{4}$

\subsection{Eigen frequency of the $\mathrm{SJ}$ actuator}

This kind of actuator can be not connected to the signal generator and the Eigen frequency cannot be determined experimentally (such as actuator frequency characteristic determination in [6]). This SJ actuator is directly connected to the power source where voltage and current values were set and thereby the SJ actuator working frequency is adjusted automatically. 
The frequency was determined from data obtained when the actuator worked by voltage of $5 \mathrm{~V}$ and current of $0.2 \mathrm{~A}$. The measured signal was interlaced with function $y=A+B \sin (2 \cdot \pi \cdot C+D)$, where $C$ corresponds to the working frequency of $59 \mathrm{~Hz}$, [7].

\subsection{Velocity measurement}

First experiment was focused to determine the effect of synthetic jet, because it was observed that an SJ far enough from the orifice has a character of a conventional steady jet. This is caused by the development and dissipation of vortexes. This experiment was carried out for voltage of $5 \mathrm{~V}$ and current of $0.2 \mathrm{~A}$. Figure 2 demonstrates the dependence of the phase-averaged velocity $U_{\mathrm{P}}$ in a different instant of the period and the time-mean velocity $U$ on the distance from the actuator orifice No. 1. There was a visible decrease in velocity as the distance increased along $y$ direction. At a distance of $y=30 \mathrm{~mm}$, the flow oscillation practically disappeared, and the flow can be considered as a steady jet flow. $t / T=0.25$ represents phase maximum extrusion, $t / T=0.75$ phase of maximum suction, respectively. Negative values of curves $t / T=0.75$ and 0 in the orifice vicinity represents suction into the actuator cavity.

Dependence of the velocity distribution on time period in different distances from the SJ actuator orifice No. 1 shows figure 3 . On this figure is also visible that the actuator works as the synthetic jet actuator until distance of approx. $30 \mathrm{~mm}$ above the orifice. According results plotted in figures 2 and 3, the next experiments were performed in distances $(0-30) \mathrm{mm}$ from the actuator orifice.

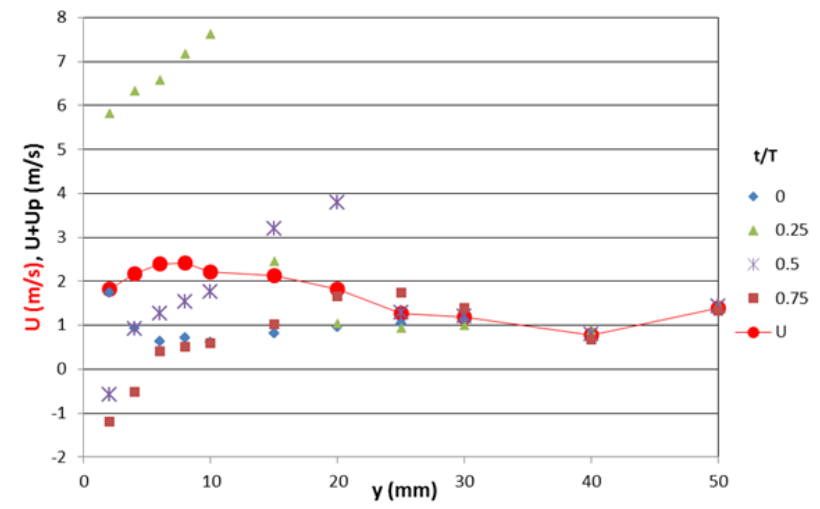

Figure 2. Dependence of the phase-averaged velocity magnitude at different instances in the period and of the time-mean velocity on the distance from the actuator orifice, orifice part No. 1, measured at $5 \mathrm{~V}, 0.2 \mathrm{~A}$.

Due to the geometry of the SJ actuator, its symmetry was supposed. For the confirmation, the experiments presented in figure 4 were performed - the velocity distribution depending on the distance from the actuator orifices. The value of voltage and current was kept on $5 \mathrm{~V}$ and $0.2 \mathrm{~A}$. The results demonstrated in figure 4 the assumption of the actuator symmetry does not confirmed. As it is visible, the shape of the velocity curves is very similar, but the values are different in range of approx. $20 \%-35 \%$, respectively. The results show that all parts of orifices do not work with the same power. This difference could be also caused with small changes of the electric power delivered into the actuator. But this power change was very small (in order of percent), so the actuator geometry can be supposed as non-symmetrical: small differences in the inner geometry, not identical geometry of the orifices, etc.

Figure 5 demonstrates the time mean velocity profiles in $x$ axes measured in different distances $y$ from the actuator orifice. With increasing distance along $y$, the velocity profile starts to be symmetrical in $x$ direction. Near the orifice, $y=(0-5) \mathrm{mm}$, there is evident a role of influence of the orifice's shape and geometry.

Velocity profiles development during the time period in distance $y=10 \mathrm{~mm}$ demonstrates figure 6 . There is visible that the vortexes of $\mathrm{SJ}$ are visible also in the distance $\pm 6 \mathrm{~mm}$ from the axis of the orifice.

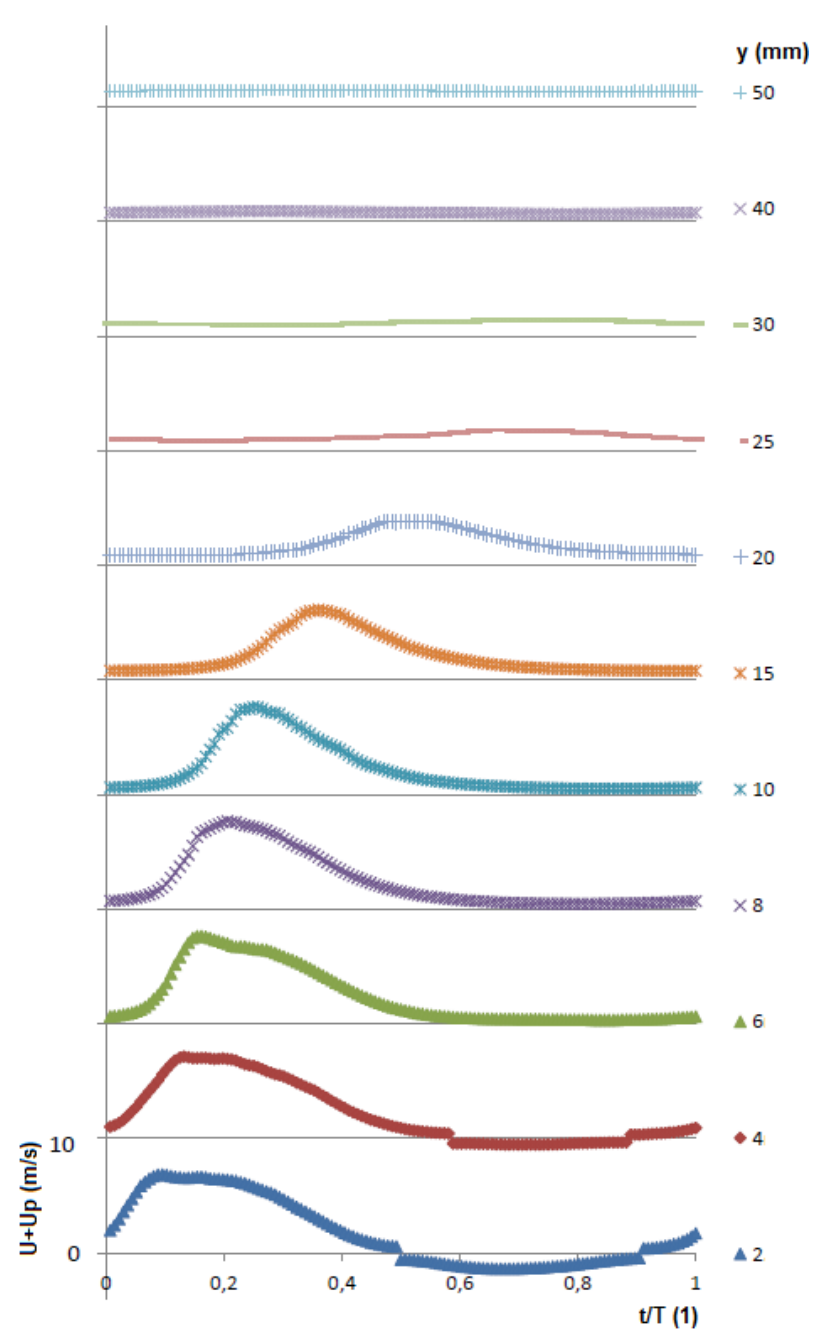

Figure 3. Phase-averaged velocity during the time period for different distances from the SJ actuator orifice, orifice part No. 1, measured at $5 \mathrm{~V}, 0.2 \mathrm{~A}$. 


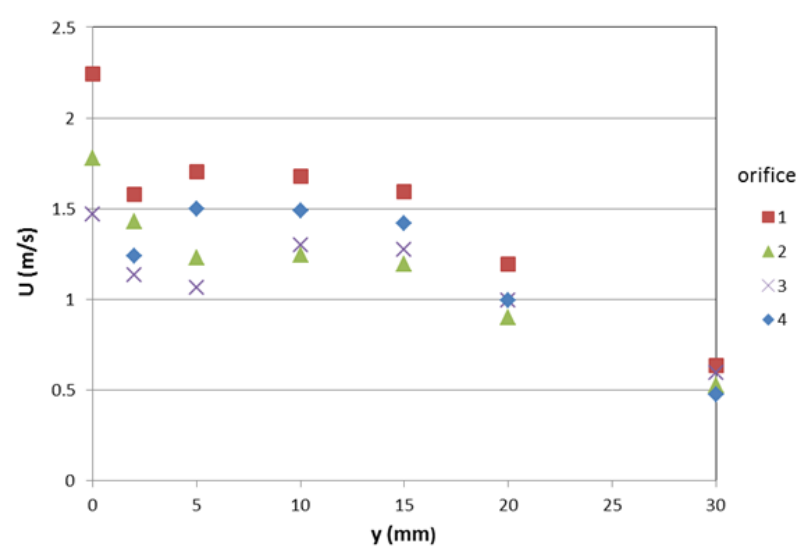

Figure 4. Time mean velocity distribution depending on the distance from the orifice, measured at $5 \mathrm{~V}, 0.2 \mathrm{~A}$.

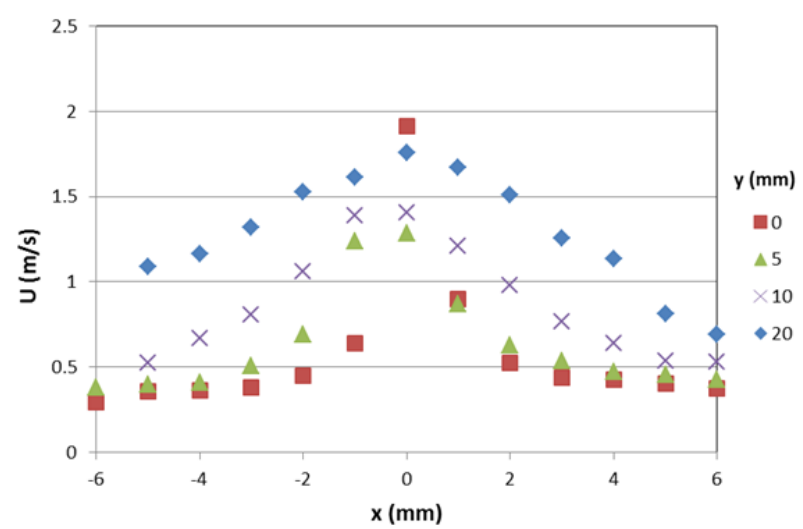

Figure 5. Time mean velocity profiles in different distances from the orifice, measured on orifice part No. 1, measured at $5 \mathrm{~V}, 0.2 \mathrm{~A}$.

\section{Discussions}

The experiments performed on the commercial synthetic jet actuator from the company Nuventix; model SynJet ZFlow 90 Outdoor Cooler were described in this paper. Experiments were carried out using thermoanemometry method in the constant temperature mode. The dependence of the phase-averaged and time mean velocity on the distance from the orifice ( $y$ direction) were investigated. Time mean velocity profile distances from the orifice have been also described in this paper.

During the experiments small non-symmetry of the SJ actuator were detected.

All experiments were carried out with voltage of $5 \mathrm{~V}$ and current of $0.2 \mathrm{~A}$; keeping of these values were monitored during the experiments.

In the next step of the experiments the changing of the electric power will be investigated, for these changes the working frequency will be determined. Also the cooling properties of this actuator will be studied.

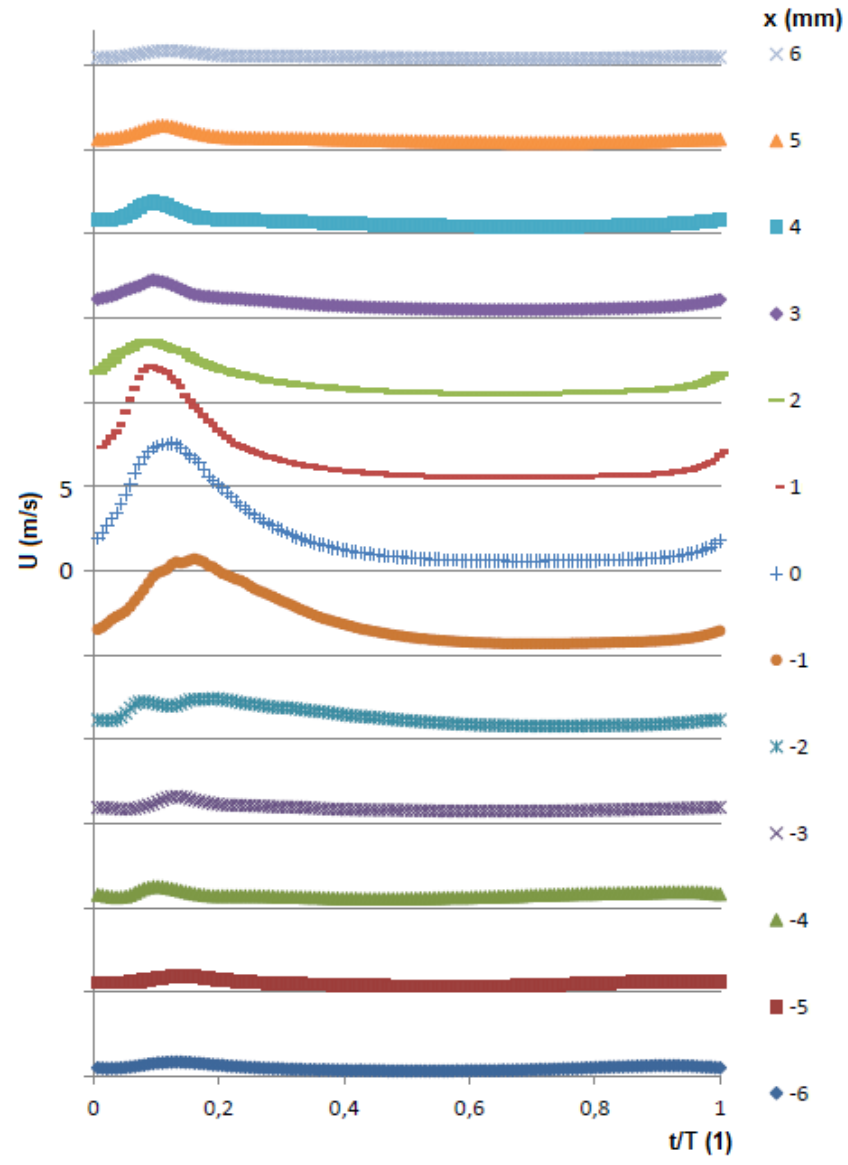

Figure 6. Phase-averaged velocity development during the time period for distance $y=10 \mathrm{~mm}$ from the SJ actuator orifice, orifice part No. 1, measured at $5 \mathrm{~V}, 0.2 \mathrm{~A}$.

\section{References}

1. http://www.aavid.com/sites/default/files/products/led /Aavid-ZFlow90-Outdoor-SynJet-April2015.pdf (cited 10/10/2015)

2. B.L. Smith, A. Glezer, Phys. Fluids 10, 2281 (1198)

3. J.E. Cater, J. Soria, J. Fluid Mech. 472, 167 (2002)

4. A. Glezer, M. Amitay, Annu. Rev. Fluid Mech. 34, 503 (2002)

5. H.H. Bruun, Hot wire anemometry (Oxford Univ. Press, 1995)

6. P. Dančová, Ph.D. thesis (Technical university of Liberec, 2012)

7. M. Šimko, Bachelor thesis (Technical university of Liberec, 2015)

\section{Acknowledgements}

We gratefully acknowledge the support of the Grant Agency of the Czech Republic - Czech Science Foundation (project no. 14-08888S). 